\title{
Biological Imaging at High Spatiotemporal Resolution
}

\author{
Hari Shroff ${ }^{1}$
}

\section{Section on High Resolution Optical Imaging, NIBIB/NIH, Bethesda, USA}

I will discuss our latest attempts to improve fluorescence microscopy techniques for following live biological phenomena at high spatiotemporal resolution. Emphasis will be given to structured illumination microscopy (SIM) and light-sheet microscopy (LSFM) techniques.

$\mathrm{SIM}^{1}$ doubles the spatial resolution of light microscopy, requiring lower light intensities and acquisition times than other super-resolution techniques. I will present SIM implementations that enable resolution doubling in live volumes $>10-20 \mathrm{x}$ thicker $^{2-4}$ than possible with conventional SIM, as well as hardware modifications that enable effectively 'instant' SIM $^{3,5}$ imaging at rates 10-100x faster than other SIM. New applications of instant SIM, including combination with total internal reflection (TIRF) and with adaptive optics ${ }^{6}$ will also be discussed.

The second half of the talk will focus on the development of inverted selective plane illumination microscopy (iSPIM), and subsequent application to the noninvasive study of neurodevelopment in nematodes ${ }^{7}$. I will discuss progress that quadruples the axial resolution of iSPIM by utilizing a second specimen view, thus enabling imaging with isotropic spatial resolution (dual-view iSPIM, or diSPIM ${ }^{8,9}$ ). Newer multiview results with more objectives ${ }^{10}$ and more views ${ }^{11}$, further improving spatial resolution, will also be shown. Applications of these technologies will be presented, including computational methods for untwisting worm embryos ${ }^{12}$, with the goal of building a neurodevelopmental atlas with subcellular resolution ${ }^{13}$.

Time permitting, I will also discuss 3 new and unpublished research projects in my lab: (1) rapid, sensor-less adaptive optics for use in SIM and LSFM; (2) 3D orientation sensing via multi-view fluorescence polarization; (3) structured illumination microcopy with $\sim 5$-fold improvement in axial resolution.

\section{References:}

1 Gustafsson, M. G. L. Surpassing the lateral resolution limit by a factor of two using structured illumination microscopy. J. Microsc. 198, 82-87 (2000).

2 York, A. G. et al. Resolution Doubling in Live, Multicellular Organisms via Multifocal Structured Illumination Microscopy. Nature Methods 9, 749-754 (2012).

3 Winter, P. W. et al. Two-photon instant structured illumination microscopy improves the depth penetration of superresolution imaging in thick scattering samples. Optica 1, 181-191 (2014).

4 Winter, P. W. et al. Incoherent structured illumination improves optical sectioning and contrast in multiphoton superresolution microscopy. Optics Express 23, 5327-5334 (2015).

5 York, A. G. et al. Instant super-resolution imaging in live cells and embryos via analog image processing. Nat Methods 10, 1122-1126 (2013).

6 Zheng, W. et al. Adaptive optics improves multiphoton super-resolution imaging. Nat Methods 14, 869-872 (2017).

$7 \mathrm{Wu}, \mathrm{Y}$. et al. Inverted selective plane illumination microscopy (iSPIM) enables coupled cell identity lineaging and neurodevelopmental imaging in Caenorhabditis elegans. Proc. Natl. Acad. Sci. USA 108, 17708-17713 (2011).

$8 \mathrm{Wu}, \mathrm{Y}$. et al. Spatially isotropic four-dimensional imaging with dual-view plane illumination microscopy. Nat Biotechnol. 31, 1032-1038 (2013). 
9 Kumar, A. et al. Dual-view plane illumination microscopy for rapid and spatially isotropic imaging. Nature Protocols 9 , 2555-2573 (2014).

$10 \mathrm{Wu}, \mathrm{Y}$. et al. Simultaneous multiview capture and fusion improves spatial resolution in wide-field and light-sheet microscopy. Optica 3, 897-910 (2016).

$11 \mathrm{Wu}, \mathrm{Y}$. et al. Reflective imaging improves spatiotemporal resolution and collection efficiency in light sheet microscopy. Nat Commun. 8, 1452 (2017).

12 Christensen, R. et al. Untwisting the Caenorhabditis elegans embryo. eLife, e10070 (2015).

13 Santella, A. et al. WormGUIDES: an interactive single cell developmental atlas and tool for collaborative multidimensional data exploration. . BMC Bioinformatics 16, 1-9 (2015).

14 The author acknowledges funding from the National Institute of Biomedical Imaging and Bioengineering and the National Institutes of Health. 